\title{
Efficient Physics-Based Tracking of Heart Surface Motion for Beating Heart Surgery Robotic Systems
}

\author{
Evgeniya Bogatyrenko · Pascal Pompey · Uwe D. Hanebeck
}

Received: 17.01.2010 / Accepted: date

\begin{abstract}
Purpose Tracking of beating heart motion in a robotic surgery system is required for complex cardiovascular interventions.

Methods A heart surface motion tracking method is developed, including a stochastic physics-based heart surface model and an efficient reconstruction algorithm. The algorithm uses the constraints provided by the model that exploits the physical characteristics of the heart. The main advantage of the model is that it is more realistic than most standard heart models. Additionally, no explicit matching between the measurements and the model is required. The application of meshless methods significantly reduces the complexity of physics-based tracking.

Results Based on the stochastic physical model of the heart surface, this approach considers the motion of the intervention area and is robust to occlusions and reflections. The tracking algorithm is evaluated in simulations and experiments on an artificial heart. Providing higher accuracy than the standard model-based methods, it copes successfully with occlusions and provides high performance when all measurements are not available.

Conclusions Combining the physical and stochastic description of the heart surface motion ensures physically correct and accurate prediction. Automatic initialization of the physics-based cardiac motion tracking enables system evaluation in a clinical environment.
\end{abstract}

Keywords beating heart surgery · tracking - model-based estimation · trinocular camera system · computer vision

Evgeniya Bogatyrenko · Pascal Pompey · Uwe D. Hanebeck Intelligent Sensor-Actuator-Systems Laboratory (ISAS),

Institute for Anthropomatics,

Karlsruhe Institute of Technology (KIT), Germany.

Tel.: +49-721-608-4354, +49-721-608-3909

E-mail: bogatyrenko@kit.edu, Uwe.Hanebeck@ieee.org

\section{Introduction}

In comparison to traditional on-pump procedures, off-pump procedures, or so called beating heart surgery, reduce the risk of complications associated with temporarily stopping the heart during surgery [1]. However, beating heart operations are still challenging tasks for surgeons. They put high demands on the synchronization of surgical instruments with the heart motion [2] and require high concentration and long experience from a surgeon. Manual tracking of the complex motion of the beating heart cannot be achieved by a surgeon without errors, because heart motion exceeds the human tracking bandwidth [3]. The application of a robotic surgery system to such medical interventions can overcome this limitation. Further benefits include high accuracy, fine manipulation capability, good repeatability, high reliability, and lack of fatigue.

\subsection{Robotic Surgery System}

The challenges of the operation on a beating heart, such as the simultaneous visual and action coordination, will be taken over by the robotic surgery system, which is depicted in Fig. 1. It consists of a measurement unit, such as cameras or other sensors, an instrument robot, a controller, and an image stabilization unit.

Because of contact-free measurements and observation of the whole intervention area, a camera system is a practical way to retrieve three-dimensional information about the heart movement. It can be installed over the operation table for open thorax surgery or integrated in an endoscope for minimally invasive surgery. The measurements provided by this system are used for estimation and prediction of the heart motion. The instrument robot that synchronizes the surgical instruments with the heart motion uses these measurements as a feedback signal. The surgeon directly con- 


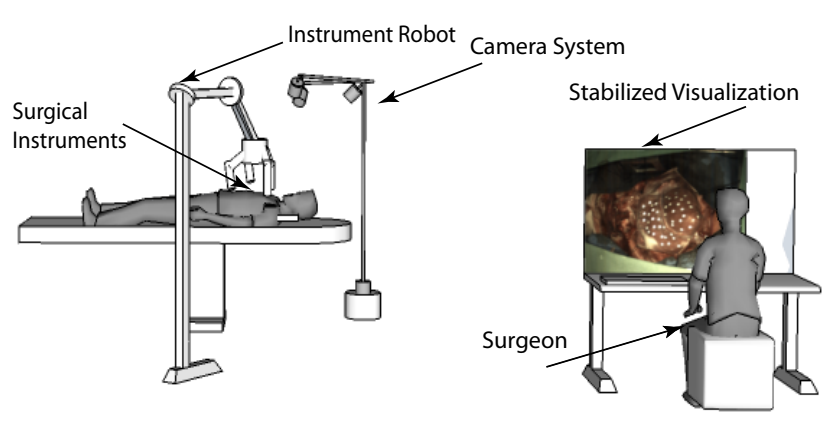

Fig. 1 Beating heart surgery robotic system, which allows the surgeon to perform beating heart operations.

trols the instrument robot and a stabilized visualization of the surgical scene gives him an impression of operating on a non-beating heart. Stabilized visualization and an automatic synchronization of surgical instruments with the beating heart will assist a surgeon by operation on a beating heart.

The main requirements on a robotic system for beating heart surgery are: high accuracy, reliability, adaptation to any patient, and real-time operability.

The accuracy of the system should range from $100 \mu \mathrm{m}$ to $300 \mu \mathrm{m}$. This is determined by the diameter of coronary arteries, which range from $2 \mathrm{~mm}$ to smaller than $0.5 \mathrm{~mm}$, as stated in [4]. The real-time operability requires that the processing time should be low for a precise estimation of the heart motion. As the dominant mode of the heart motion is on the order of $1-2 \mathrm{~Hz}$, data processing at $4 \mathrm{~Hz}(250 \mathrm{~ms})$ is sufficient, according to the Nyquist-Shannon sampling theorem [5]. However, a spectral analysis of the beating heart motion shows frequencies up to $15 \mathrm{~Hz}$. The heart motion is not perfectly periodic and therefore, a high acquisition speed is necessary for sufficiently capturing the heart dynamics. In order to estimate the beating heart motion, a method for efficient and accurate reconstruction and robust tracking of the heart surface is of significant importance. Nevertheless, this is still a challenging task due to the heart's complex dynamics and non-reliable noisy measurements.

\subsection{Related Work}

Existing approaches for tracking the beating heart motion are analyzed according to the following criteria: reconstruction accuracy and constraints on the heart movement.

The feasibility of vision-based systems for the compensation of the heart motion is first explored in [6], where two high-speed cameras are used to track artificial landmarks fixed on a heart. However, this method is only applied for 2D tracking without extraction of depth information. Tracking the heart motion in stereo endoscopic beating heart sequences is proposed in [7-10]. However, these approaches are sensitive to visual artifacts and variations in illumination. Furthermore, they fails in cases of occlusions and areas of low-texture, and provides depth information of limited accuracy. Although the extraction of the visual information is facilitated by introducing artificial markers [11] or template matching with texture analysis $[12,13]$, the accuracy of the tracking needs improvement.

Up to now, most tracking approaches apply constraints on the heart movement. These methods $[14,15]$ deal with the tracking of an intervention point on the heart surface without taking into account the movement of the intervention area. The implications of such a constraint are low accuracy and high demands on the reaction time of the surgical system. The reason for that is a deficiency of the information about movement of the points near the intervention point. Therefore, the observation of the whole intervention area during tracking allows to reduce the high demands on the reaction time of the surgical system. It is essential in case of the simultaneous synchronization of several instruments with the motion of different points in this area. The motion of the intervention area is exploited in [16], where a parametric thin-plate spline model for approximation of the heart surface is proposed. This model is geometric and assumes a small inter-frame motion. By ignoring the physical information about the heart, it can lead to a physically incorrect estimation of the heart motion.

In [17], a physical heart surface model in the form of a thin membrane is proposed for intraoperative tracking of the heart motion. The motion in consideration is greatly simplified by assuming that the heart moves only in out-of-plane direction. In [18], the heart surface is efficiently modeled as a thin plate. However, the model's presumption of a small out-of-plane deflection can only be applied for operations on a stabilized beating heart. In both methods, the initialization of the model geometry and parameters is not considered. The detection of the model geometry is complicated, as the heart moves over consecutive image sequence. Furthermore, the heart geometry and parameters vary from patient to patient. Another difficulty of employing a physical model is that the modeling of the complex heart motion is computationally expensive and hardly possible in real-time. The first reason for that is the usage of complex geometric models, such as models for a preoperative planning or a therapy testing $[19,20]$. Another reason, the numerical solution of the partial differential equations (PDE) with traditional methods, such as the finite-element method (FEM), generates system-related response delays.

\subsection{Key Idea}

In this paper, a physics-based heart motion tracking method is proposed for application in robotic systems for beating 


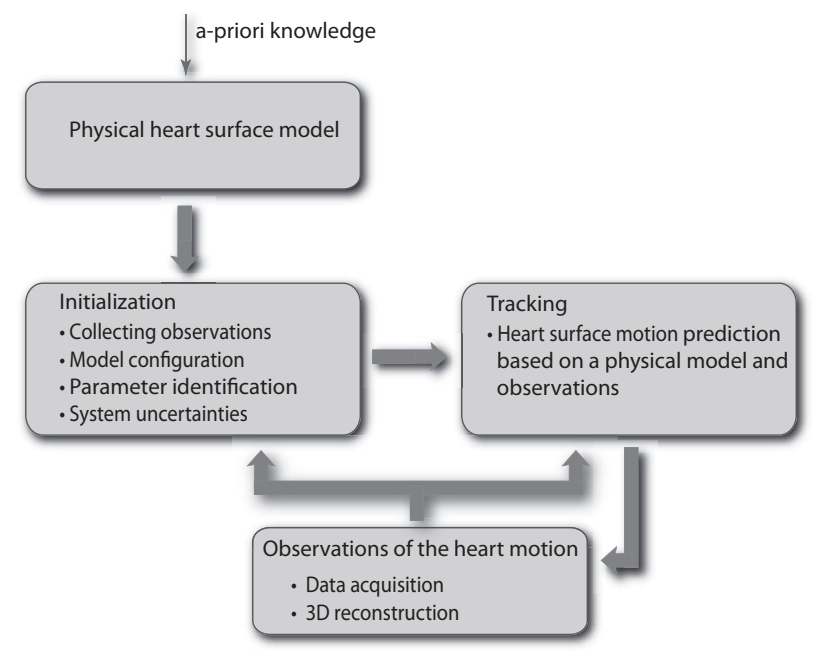

Fig. 2 General concept of the physics-based tracking system.

heart surgery. It incorporates a physical heart surface model and an efficient reconstruction algorithm.

The principle advantage of the physical heart surface model is that no constraints on the type of the heart motion are made. For example, there are no assumptions on periodicity or small out-of-plane movement. The automatic calibration of the model based on camera observations allows to determine the model geometry and parameters before a beating heart operation. Finally, due to application of meshless methods, the computational complexity of the heart surface model is significantly reduced.

Another difference of the physics-based tracking method from other tracking approaches is the reconstruction algorithm. For improving the efficiency of the reconstruction, the correspondence analysis employs a set of selection criteria. The correspondences in camera images and between reconstructed measurement points are thereby constrained by the physics-based prediction. A trinocular camera setup allows to reduce the complexity of the correspondence problem.

By exploiting the information about physical characteristics of the heart, the physics-based tracking ensures a physically correct and more accurate prediction in comparison to the standard model-based methods, as shown in experimental evaluation in Section 7.1. Consideration of the stochastic uncertainties of the model and measurement system allows thereby to evaluate the prediction quality and increase the accuracy of the tracking. According to the experimental results in Section 7.2.3, the proposed physics-based tracking is robust, since it copes with the total occlusions, when the heart surface is completely covered. In this case, the conventional interpolation methods cannot be applied, because no measurement information is available.

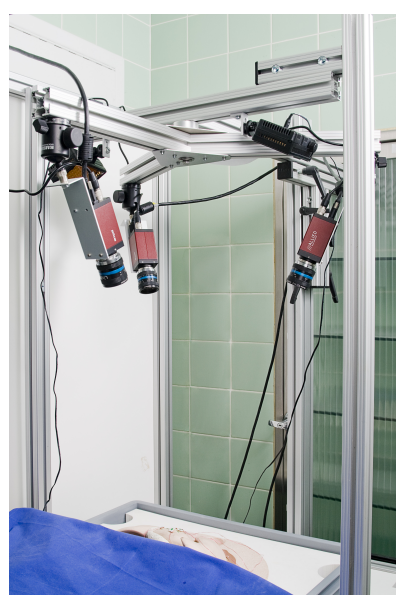

Fig. 3 Trinocular camera setup.

\section{System Overview}

This section gives an overview of the proposed tracking system. The general system concept is presented in the Fig. 2. By taking a modular, compositional view of the system design problem, we introduce the components of the physicsbased tracking approach: a physical heart surface model, an initialization procedure, tracking, and observations. The modular composition of the system allows for each component to work independently.

A physical heart surface model exploiting the a-priori information about physical characteristics of the heart will be presented in Section 3.1. The specialty of the proposed model is that only the intervention area of the heart surface is considered. By describing the heart surface as a physical elastic body in form of PDE, we employ meshless methods for the decomposition of the PDE. The obvious advantage of meshless methods, in comparison to traditional numerical methods, such as FEM, finite differences (FDM), or finite volume methods (FVM), is that no information on the relationship between the nodes is required and thus no element mesh is needed. Since meshless discretization techniques are only based on a set of points, they are better suited to handle strong deformations.

The initialization procedure described in Section 5 is very important for adaptation of the heart surface model to every patient. It opens up possibilities for evaluation of the physics-based tracking approach in a clinical environment. In order to achieve an accurate estimation, the heart surface model is calibrated based on the observations. The model parameters are calibrated according to their physical constraints. The observations contain information about the 3D position of the heart landmarks, which is extracted from camera measurements. After collecting the observations by means of a measurement-based tracking approach, the model geometry, parameters and uncertainties of the system are identified. 
Further, the heart kinematics are tracked over consecutive image frames as presented in Section 6. For tracking, the position of the heart landmarks is predicted by means of an estimation approach combining model and measurement information. The best estimate of the heart surface kinematics, such as deflection and velocity, is provided in this way.

For achieving high accuracy and reliability of the tracking system, robust and precise observation is essential. In this paper, we propose a trinocular camera setup for data acquisition, shown in Fig. 3. This setup leads to a robust reconstruction and provides more information about the operation area. The proposed surround orientation of the cameras ensures nearly the same uncertainties in all directions. In this case, most of the optical rays are almost orthogonal. This allows a better elimination of artifacts due to the socalled transfer property that facilitates the establishing correspondences over three views [21]. Given a point or line correspondence over two views, the position in the third view is determined. As a result, the trinocular camera system increases reliability of the whole reconstruction process by reducing outliers [21,22]. Although the proposed camera setup is only applicable for open thorax surgery, the trinocular camera system can also be integrated in an endoscope for minimally invasive interventions. For reducing the computational time, the extraction of the measurement information is provided by a feature-based image processing algorithm. Artificial passive colored markers are attached to the heart surface in order to simplify the feature extraction. In real environment conditions, the markers can be spread over the intervention area. They cling tightly to the heart surface, as shown in Fig. 4. For the extraction of the image information, a standard color-based segmentation algorithm is used. The detected colored area, satisfying the size criteria of the heart landmark, is approximated by a circle. The center of a circle is assumed to represent a measurement point. Instead of artificial markers, natural landmarks or image features can be used for the reconstruction of the 3D motion by the proposed reconstruction algorithm.

By employing the predicted heart position for identification of the measurement points over consecutive image frames, we increase the efficiency of the reconstruction. By introducing some selection procedures on three camera images and on reconstructed measurement points, as presented in Section 4.2, we sufficiently reduce the computational time and ensure the consistency of the returned solution. For avoiding the ambiguities in correspondence analysis, only the points detected in all three cameras are reconstructed.

In the next sections, the main components of the physicsbased tracking approach are described in detail. These components include: 3D reconstruction, a physical heart surface model, calibration and tracking.

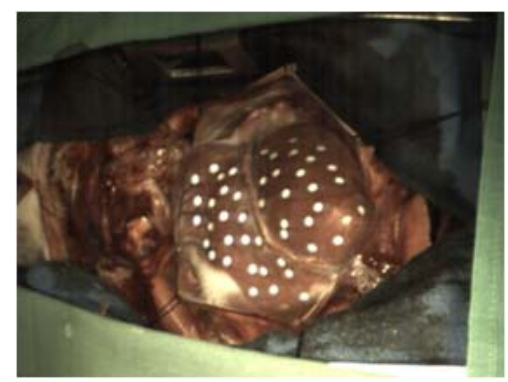

Fig. 4 Heart with attached landmarks, which are arbitrarily distributed on the heart surface.

\section{Heart Surface Modeling}

A heart surface model considering the physical characteristics of the heart is proposed in this section. Starting with the description of the heart surface as a distributed parameter system (DPS) in the form of stochastic PDEs, we derive a state-space form by means of spatial and temporal decomposition. The stochastic uncertainties corrupting the system and measurements are thereby considered.

\subsection{Physical Heart Surface Model}

The heart surface is assumed to have the behavior of a linear elastic physical body with isotropic material structure. Because only a small area of the stabilized heart surface is considered, as shown in Fig. 5, this assumption is justified. The undeformed configuration of this body is defined by the material coordinates $\underline{x}=\left[\underline{x}^{\mathrm{T}}, y^{\mathrm{T}}, \underline{z}^{\mathrm{T}}\right]^{\mathrm{T}} \in \mathbb{R}^{3 N}$ in a domain $\Omega \in \mathbb{R}^{N}$ enclosed by Neumann $\partial \Omega_{N}$ and Robin boundary conditions $\partial \Omega_{R}$, so that $\partial \Omega=\partial \Omega_{N} \cup \partial \Omega_{R}$. The displacement field is specified as a continuous mapping $\underline{u}=\left[\underline{u}_{x}^{\mathrm{T}}, \underline{u}_{y}^{\mathrm{T}}, \underline{u}_{z}^{\mathrm{T}}\right]^{\mathrm{T}}: \mathbb{R}^{3 N} \rightarrow \mathbb{R}^{3 N}$, which defines for each point $\underline{x}$ in material coordinates a corresponding point in world coordinates where the heart surface is displaced. The motion of the heart surface is formulated as a boundary and initial value problem. The strong mathematical model is described by a set of stochastic PDEs, written in a concise matrix form

$$
\mathbf{L}^{\mathrm{T}} \underline{\boldsymbol{\sigma}}(\underline{u}(\underline{x}, t))+\underline{\boldsymbol{b}}(\underline{x}, t)-\rho \underline{\ddot{\boldsymbol{u}}}(\underline{x}, t)-\eta \underline{\dot{\boldsymbol{u}}}(\underline{x}, t)=\underline{R}_{I}(\underline{x}, t)=\underline{0},
$$

where $\mathbf{L} \in \mathbb{R}^{6 N \times 3 N}$ is the differential operator matrix given by

$\mathbf{L}=\left[\begin{array}{ccc}\frac{\partial}{\partial x} & 0 & 0 \\ 0 & \frac{\partial}{\partial y} & 0 \\ 0 & 0 & \frac{\partial}{\partial z} \\ 0 & \frac{\partial}{\partial z} & \frac{\partial}{\partial y} \\ \frac{\partial}{\partial z} & 0 & \frac{\partial}{\partial x} \\ \frac{\partial}{\partial y} & \frac{\partial}{\partial x} & 0\end{array}\right]$ 


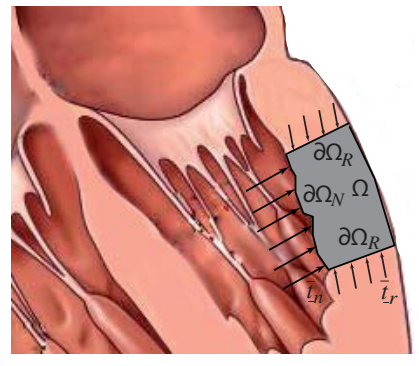

Fig. 5 Physics-based heart surface model (side view) describing the heart wall inside of the intervention area as a physical elastic body.

Here the random vector $\underline{\boldsymbol{u}}$ represents the distributed displacement of the heart surface. The internal heart forces are defined by a distributed load $\underline{\boldsymbol{b}}=\left[\underline{\boldsymbol{b}}_{x}^{\mathrm{T}}, \underline{\boldsymbol{b}}_{y}^{\mathrm{T}}, \underline{\boldsymbol{b}}_{z}^{\mathrm{T}}\right]^{\mathrm{T}}$ in the $x, y$ and $z$ directions, respectively. The vector

$\underline{\boldsymbol{\sigma}}(\underline{u}(\underline{x}, t))=\left[\underline{\boldsymbol{\sigma}}_{x x}^{\mathrm{T}}, \underline{\boldsymbol{\sigma}}_{y y}^{\mathrm{T}}, \underline{\boldsymbol{\sigma}}_{z z}^{\mathrm{T}}, \underline{\boldsymbol{\sigma}}_{y z}^{\mathrm{T}}, \underline{\boldsymbol{\sigma}}_{x z}^{\mathrm{T}}, \underline{\boldsymbol{\sigma}}_{x y}^{\mathrm{T}}\right]^{\mathrm{T}} \in \mathbb{R}^{6 N}$

is the stress tensor with six independent stress components. The parameter $\rho$ describes the density and the parameter $\eta$ stays for the damping coefficient of the elastic material. The natural or Neumann boundary conditions are imposed in the domain $\partial \Omega_{N}$ by

$\mathbf{N} \underline{\boldsymbol{\sigma}}(\underline{u}(\underline{x}, t))=\underline{\overline{\boldsymbol{t}}}_{n}(\underline{x}, t)$,

where $\mathbf{N} \in \mathbb{R}^{6 N \times 3 N}$ is the matrix containing an outward normal vector on the natural boundary and $\underline{\bar{t}}_{n}$ is the prescribed traction force. This force is determined by atrial pressure that can be monitored in cardiac surgery by a central line catheter [23]. The mixed or Robin conditions, also called elastic foundation, are assumed to represent the heart surface motion in the domain $\partial \Omega_{R}$ by

$\alpha \mathbf{N} \underline{\boldsymbol{\sigma}}(\underline{u}(\underline{x}, t))+\beta \mathbf{R} \underline{\boldsymbol{u}}(\underline{x}, t)=\underline{\overline{\boldsymbol{t}}}_{r}(\underline{x}, t)$,

where $\mathbf{R} \in \mathbb{R}^{3 N \times 3 N}$ is the stiffness matrix and $\alpha, \beta$ are weighting coefficients. Physically, this implies that the traction force $\bar{t}_{r}$ is proportional to the boundary displacement. The initial values for displacement and velocity of the heart surface motion are defined by the reconstructed 3D information stemming from the camera measurements.

As a result, the heart surface motion is described as a distributed continuous phenomenon. Further, this DPS is converted into a lumped-parameter form, which is achieved by numerical methods for solving PDEs.

\subsection{Spatial Decomposition}

For spatial decomposition of the PDEs, the meshless collocation method [24] is applied. Without the need for a predefined mesh, this method results in higher computational efficiency than the classical methods [25]. Concerning the robotic surgery system, meshless methods can contribute to easier adaptation of the model to any patient, give flexibility in handling surgical interventions, and reduce the time for initialization of the robotic surgery system [18].

At first, the solution function $\underline{\boldsymbol{u}}$ of the PDEs given by equations (1), (2), and (3) is replaced by a finite approximation $\underline{\boldsymbol{u}}^{h}$ as

$\underline{\boldsymbol{u}}^{h}(\underline{x}, t) \approx \boldsymbol{\Phi}(\underline{x}) \underline{\boldsymbol{c}}(t)$

where the temporal dependence of the PDE solution is represented by the scaling factors $\underline{c} \in \mathbb{R}^{3 N}$. The spacial dependence is defined by the moving least square (MLS) shape functions $\boldsymbol{\Phi}(\underline{x}) \in \mathbb{R}^{3 N \times 3 N}$, as described in [25].

In order to determine the scaling factors, the method of weighted residuals can be employed. It aims at minimizing the approximation error over the solution domain $\Omega$ and therefore satisfies the condition

$\int_{\Omega} \mathbf{W}(\underline{x}) \underline{R}_{I}(\underline{x}, t) d \Omega=\underline{0}$,

for all possible test functions $W(\underline{x})$. The residual $R_{I}(\underline{x}, t)$ is defined in equation (1).

Further, we suppose that the error vanishes at certain collocation points $N$ and define the test function $W(\underline{x})=\delta\left(\underline{x}-\underline{x}_{j}\right)$ as a Dirac delta distribution. The derived in this way collocation method [26] has three major advantages in comparison to the other meshless methods. Since no integration is required, the construction of the final system of equations is efficient. Moreover, the shape functions are only evaluated at nodes rather than at integration points. Due to the fact that only the points are considered, the refinement and adaptive analysis can be realized faster than in FEM.

As a result of the spatial decomposition of the PDEs (1), (2), and (3), the set of stochastic ordinary differential equations (ODE), enforced at $N$ collocation points in the solution domain, is given by

$\mathbf{M} \underline{\ddot{c}}(t)+\mathbf{C} \underline{\dot{c}}(t)+\mathbf{K} \underline{\boldsymbol{c}}(t)+\mathbf{\Phi} \underline{\boldsymbol{p}}(t)=\underline{0}$,

where the mass matrix is defined as

$\mathbf{M}=\left[\begin{array}{c}-\rho \Phi(\underline{x}) \\ \mathbf{0} \\ \mathbf{0}\end{array}\right]$

Here the parameter $\rho$ denotes the material density of the heart surface. The last two entries of the stiffness matrix

$\mathbf{K}=\left[\begin{array}{c}\mathbf{L}^{\mathrm{T}} \mathbf{D L} \boldsymbol{\Phi}(\underline{x}) \\ \mathbf{N D L} \boldsymbol{\Phi}(\underline{x}) \\ \alpha \mathbf{N D L} \boldsymbol{\Phi}(\underline{x})+\beta \mathbf{R} \boldsymbol{\Phi}(\underline{x})\end{array}\right]$ 
represent the imposition of the boundary conditions. The material matrix

$\mathbf{D}=\left[\begin{array}{cccccc}d_{11} & d_{12} & d_{12} & 0 & 0 & 0 \\ 0 & d_{11} & d_{12} & 0 & 0 & 0 \\ 0 & 0 & d_{11} & 0 & 0 & 0 \\ 0 & 0 & 0 & \frac{d_{11}-d_{12}}{2} & 0 & 0 \\ 0 & 0 & 0 & 0 & \frac{d_{11}-d_{12}}{2} & 0 \\ 0 & 0 & 0 & 0 & 0 & \frac{d_{11}-d_{12}}{2}\end{array}\right]$

is defined by the physical parameters of the heart surface such as Young's modulus $E$ and Poisson's ratio $\nu$ as

$d_{11}=\frac{E(1-\nu)}{(1-2 \nu)(1+\nu)}, d_{12}=\frac{E \nu}{(1-2 \nu)(1+\nu)}$.

The matrix $\mathbf{N} \in \mathbb{R}^{6 N \times 3 N}$ is described by outward normals on the boundary. The matrix of the damping coefficients is determined by assuming Rayleigh damping

$\mathbf{C}=\left[\begin{array}{c}\eta_{1} \mathbf{M}+\eta_{2} \mathbf{K} \\ \mathbf{0} \\ \mathbf{0}\end{array}\right]$

where $\eta_{1}$ and $\eta_{2}$ represent the damping coefficients. Finally, the load vector is described as

$\underline{\boldsymbol{p}}(t)=\left[\underline{\boldsymbol{b}}(t)^{\mathrm{T}}, \underline{\overline{\boldsymbol{t}}}_{n}(t)^{\mathrm{T}}, \underline{\boldsymbol{t}}_{r}(t)^{\mathrm{T}}\right]^{\mathrm{T}}$.

Consequently, the spatial decomposed dynamic system defined in equation (5) is now ready to specify the time evolution leading to the time-discrete finite-dimensional system equation.

\subsection{State-Space Form}

Approaches to state estimation require a system description in a state-space form. For that purpose, the heart surface motion should be described by the discrete finite-dimensional system and measurement equations that represent the process dynamics and the observation model. In the last section, the DPS characterizing the heart dynamics was converted in a space-discrete system. In this section, for obtaining the time-discrete system, an implicit Euler discretization method is preferred, as it offers unconditional numerical stability.

The model of the process dynamics or the system equation relates the states of the heart movement at different time instances. Assuming that the system input $\boldsymbol{p}$ is nearly constant between two time instances $t=\left[t_{k}, t_{k}+1\right]$, we get the time-discrete state-space system equation

$\underline{\boldsymbol{\xi}}_{k+1}=\mathbf{A} \underline{\boldsymbol{\xi}}_{k}+\mathbf{B}\left(\underline{\hat{p}}_{k}+\underline{\boldsymbol{w}}_{k}\right)$ describing the heart surface motion, where the system matrix $\mathbf{A} \in \mathbb{R}^{6 N \times 6 N}$ and the input matrix $\mathbf{B} \in \mathbb{R}^{6 N \times L}$ are time-invariant. They are defined by

$$
\begin{aligned}
& \mathbf{A}=\left(\left[\begin{array}{cc}
\mathbf{M} & \mathbf{C}+\mathbf{K} \Delta t \\
-\mathbf{I} \Delta t & \mathbf{I}
\end{array}\right]\right)^{-1}\left[\begin{array}{cc}
\mathbf{M} \mathbf{C} \\
\mathbf{0} & \mathbf{I}
\end{array}\right], \\
& \mathbf{B}=\left(\left[\begin{array}{cc}
\mathbf{M} & \mathbf{C}+\mathbf{K} \Delta t \\
-\mathbf{I} \Delta t & \mathbf{I}
\end{array}\right]\right)^{-1}\left[\begin{array}{l}
\mathbf{\Phi} \\
\mathbf{0}
\end{array}\right] .
\end{aligned}
$$

The state vector $\underline{\boldsymbol{\xi}}=\left[\underline{\dot{\boldsymbol{c}}}(t)^{\mathrm{T}}, \underline{\boldsymbol{c}}(t)^{\mathrm{T}}\right]^{\mathrm{T}} \in \mathbb{R}^{6 N}$ describes the displacement and the velocity of the heart surface. The known system input is denoted by $\hat{p}_{k}$ at time instance $t_{k}$. The stochastic error term $\underline{\boldsymbol{w}}_{k} \sim \mathcal{N}\left(0, \mathbf{C}_{k}^{w}\right)$ is white zero-mean Gaussian noise with covariance $\mathbf{C}_{k}^{w}$.

Since the heart surface position is reconstructed from the measurements provided by a camera system, the observation model can be assumed to be linear. This model is characterized by the measurement equation that relates the obtained observation $\underline{\hat{y}}_{k}$ at time step $k$ to the state $\underline{\boldsymbol{\xi}}_{k}$. By considering the stochastic noise of a reconstruction, the observation model can be written in the form

$\underline{\hat{y}}_{k}=\mathbf{H} \underline{\boldsymbol{\xi}}_{k}+\underline{\boldsymbol{v}}_{k}$,

where the measurement matrix $\mathbf{H} \in \mathbb{R}^{L \times 6 N}$ is defined by $\mathbf{H}=[\mathbf{0} \boldsymbol{\Phi}(\underline{x})]$. The vector $\underline{\boldsymbol{v}} \sim \mathcal{N}\left(0, \mathbf{C}_{k}^{v}\right)$ denotes the white zero-mean Gaussian measurement noise with covariance $\mathbf{C}_{k}^{v}$.

As a result, the state-space model that describes the physical behavior of the heart motion is derived. However, the physics-based tracking of the heart surface motion is only possible after a system initialization. The purpose of the initialization is to match the state-space model to the observations. The observations describing the $3 \mathrm{D}$ position of the heart surface are provided by an efficient 3D reconstruction to be introduced in the next section.

\section{3D Reconstruction}

The proposed selection criteria for efficient reconstruction of the 3D information from camera images is described in this section. Our reconstruction algorithm is special because it uses selection criteria applied to the image correspondences and reconstructed measurement points, and constraints imposed by a physics-based prediction.

\subsection{Reconstruction}

A calibration of cameras provides three camera projection matrices $\mathbf{P}^{\prime} \in \mathbb{R}^{3 \times 4 N}, \mathbf{P}^{\prime \prime} \in \mathbb{R}^{3 \times 4 N}$ and $\mathbf{P}^{\prime \prime \prime} \in \mathbb{R}^{3 \times 4 N}$. The set of 3D measurement points $\underline{\hat{X}} \in \mathbb{R}^{4 N}$ conveniently represented in homogeneous coordinates are projected to the image correspondences in the sense that $\mathbf{P}^{\prime} \underline{\hat{X}}=\underline{x}^{\prime}, \mathbf{P}^{\prime \prime} \underline{\hat{X}}=\underline{x}^{\prime \prime}$ and $\mathbf{P}^{\prime \prime \prime} \underline{\hat{X}}=\underline{x}^{\prime \prime \prime}$. Thus, the 
point $\underline{\hat{X}}$ is projected to the three given $2 \mathrm{D}$ data points represented in homogeneous coordinates $\underline{x}^{\prime} \in \mathbb{R}^{3 N}, \underline{x}^{\prime \prime} \in \mathbb{R}^{3 N}$, $\underline{x}^{\prime \prime \prime} \in \mathbb{R}^{3 N}$ and can be reconstructed by solving this system of linear equations, as described in [21]. Since there are uncertainties in the camera measurements, the image correspondences do not satisfy the epipolar constraint, and thus, there will not be a point $\underline{\hat{X}}$ that exactly satisfies the given equations. To overcome this, the least squares method is used to estimate the real 3D points $\underline{\hat{X}}$.

The 3D reconstruction is possible on the basis of two views, retrieved by a binocular camera system. However, adding information from the third view reduces the effect of noise and thus, enables better reconstruction accuracy.

\subsection{Correspondence Analysis}

The task of analysis is to find a set of correspondences over the three camera images $\underline{x}^{\prime} \leftrightarrow \underline{x}^{\prime \prime} \leftrightarrow \underline{x}^{\prime \prime \prime}$ where $\underline{x}^{\prime}, \underline{x}^{\prime \prime}$ and $\underline{x}^{\prime \prime \prime}$ are projections of the same 3D point $\underline{\hat{X}}$.

The common way to identify the real image correspondences is to look for the sets of points $\underline{x}^{\prime}, \underline{x}^{\prime \prime}, \underline{x}^{\prime \prime \prime}$, which minimize the cost function

$C_{1}=\left\|\underline{X}^{\prime}-\underline{X}^{\prime \prime}\right\|+\left\|\underline{X}^{\prime \prime}-\underline{X}^{\prime \prime \prime}\right\|+\left\|\underline{X}^{\prime}-\underline{X}^{\prime \prime \prime}\right\|$,

where the 3D reconstructions $\underline{X}^{\prime}, \underline{X}^{\prime \prime}, \underline{X}^{\prime \prime \prime}$ are determined on basis of the correspondences over appropriate two camera views, so that

$$
\begin{aligned}
& \left\{\underline{x}^{\prime} \leftrightarrow \underline{x}^{\prime \prime}\right\} \mapsto \underline{X}^{\prime}, \\
& \left\{\underline{x}^{\prime \prime} \leftrightarrow \underline{x}^{\prime \prime \prime}\right\} \mapsto \underline{X}^{\prime \prime}, \\
& \left\{\underline{x}^{\prime} \leftrightarrow \underline{x}^{\prime \prime \prime}\right\} \mapsto \underline{X}^{\prime \prime \prime}
\end{aligned}
$$

If $\underline{x} \leftrightarrow \underline{x}^{\prime} \leftrightarrow \underline{x}^{\prime \prime}$ are real correspondences of the image points, the reconstructed $3 \mathrm{D}$ points are all noisy reconstructions of the same real point $\underline{\hat{X}}$ and thus they all lie close to $\underline{\hat{X}}$.

Though providing a robust way for finding the real correspondences, this cost function is computationally demanding because it requires solving three different least square systems. Therefore, the set of selection procedures is proposed. In this case the number of the potential good correspondences is reduced. This way, it is ensured that no conflicting solutions are returned after the correspondence analysis.

The first selection procedure for reducing the set of the potential correspondences is based on the epipolar geometry and defines a necessary condition for points to correspond. To each point in one image, there exists a corresponding epipolar line in the other image. The alignment of the cameras in the proposed system ensures that the epipolar lines of two different camera views will cross in the third view. The intersection of the epipolar lines defines the position of the third correspondence. This condition is represented by the cost function

$C_{2}=\left\|\underline{l}^{\prime} \times \underline{l}^{\prime \prime}-\underline{x}^{\prime \prime \prime}\right\|+\left\|\underline{l}^{\prime \prime} \times \underline{l}^{\prime \prime \prime}-\underline{x}^{\prime}\right\|+\left\|\underline{l}^{\prime} \times \underline{l}^{\prime \prime \prime}-\underline{x}^{\prime \prime}\right\|$,

where the vector product of epipolar lines $\underline{l}, \underline{l}^{\prime}, \underline{l}^{\prime \prime}$ defines their intersection. Selecting only the sets of points $\underline{x}^{\prime}, \underline{x}^{\prime \prime}$, $\underline{x}^{\prime \prime \prime}$, satisfying the condition, a subset of possible correspondences containing all the real correspondences is provided. In the chosen subset, conflicts can occur when one 2D point is used in two different potential correspondences. In this case, one of the two potential correspondences is necessarily false and the cost function $C_{1}$ is used to decide which one is the real correspondence and reject the other false possibility.

The next selection procedure constrains the search of possible correspondences only to the measurement points near the position of the heart landmarks predicted by physicsbased estimation. This procedure is introduced in Section 6 . For that, the predicted positions are projected back in each camera view by using the equations given in Section 4. If the image correspondences $\underline{x}^{\prime}, \underline{x}^{\prime \prime}, \underline{x}^{\prime \prime \prime}$ are in the uncertainty region of the predicted point, they are accepted as potential correspondences.

Resulting from the consequent application of these selection procedures, the correspondence analysis using a trinocular system is fast and robust while ensuring the consistency of the returned solution.

\section{Initialization}

Initialization of the physics-based tracking approach is a challenging task because of the continuous movement of the beating heart. The initialization process should automatically detect the model geometry and the parameters depending on the characteristics of the patient's heart and an intervention area. In order to initialize the model geometry, the reference positions of the measurement points on the heart surface have to be determined. For this purpose, these points have to be tracked over some periods of the heart movement in spite of noisy measurements and possible artifacts. A measurement-based tracking algorithm is proposed in the next section. The initialization of the state space model is presented in Section 5.2.

\subsection{Measurement-Based Tracking}

The measurement-based 2D tracking runs in parallel in all three camera views. The images of the measurement points are tracked under the assumption of high temporal resolution of the image stream, so that changes between two subsequent images are sufficiently small. A nearest neighbor search algorithm is applied to select sets of potential tracks in each camera view. In order to identify the real 

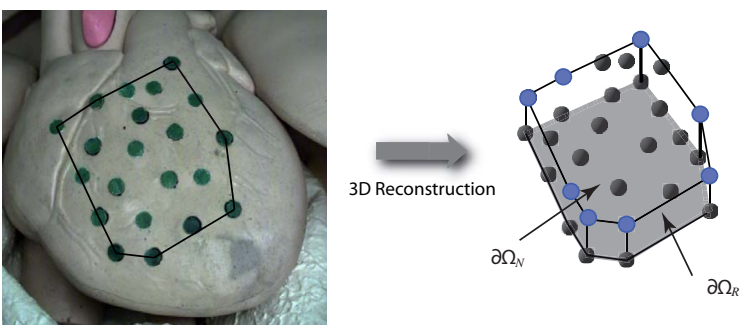

Fig. 6 Configuration of the heart surface model. The shaded region illustrates the Neumann $\partial \Omega_{N}$ boundary conditions. The Robin $\partial \Omega_{R}$ boundary conditions are imposed on the sides of the model. The convex hull defined by the blue shaded points specifies the size of the intervention area

tracks within the selected sets, the correspondence analysis described in Section 4.2 is employed. This tracking method is computationally inexpensive because it reduces the sets of points used for the computation of the correspondence analysis. Furthermore, the artifacts are filtered out by considering the tracking information in combination with the correspondence analysis. However, this method is very sensitive to temporal resolution, reflections, and occlusions and thus, is not suitable for tracking over a long period of time. Therefore, an important ability of the tracking system is that the measurement points lost during the initialization, can also be initialized during the physics-based tracking. For that, the measurement-based tracking runs in parallel to the physics-based tracking in order to determine the reference position of the non-initialized measurement points. These points can be dynamically initialized and further considered by the model according to equation (4). This flexibility allows the addition and replacement of measurement points during an operation involving a surgical system.

\subsection{Model Configuration}

The points that have been successfully tracked the whole initialization phase are identified as the measurement points on the heart surface. Their reference position is defined as the average value over all initialization time steps and is used for a configuration of the heart surface model, as presented in Fig. 6. The convex hull of these points specifies the size of the intervention area. The landmarks on the border of the intervention area define the model nodes $\partial \Omega_{R}$, where the Robin boundary conditions given in equation (3) should be imposed. The measured heart pressure characterizes the traction force in equation (2), imposed on the Neumann boundary nodes $\partial \Omega_{N}$ and the distributed load $\underline{b}$ in equation (1).

It should be noted, that the geometry of the heart surface model is dependent on the number of detected heart landmarks. Generally, at least three landmarks defining the border of the intervention area can reproduce the geometry of the intervention area. However, the more landmarks de- tected, the higher the accuracy of the model. The initialization of the model geometry is advantageous since it allows to avoid an explicit matching between the model and the observations.

As a result, the heart surface model geometry is automatically initialized involving the information provided by the initialization phase.

\subsection{Parameter Identification}

For identification of the model parameters we make use of observations $\underline{\hat{y}}_{k}$ of the heart surface motion over $N$ time instances of a initialization time interval. For fitting the heart model to the measurement data, the weighted least-squares approach [27] is applied. It minimizes the output-error criterion

$\underline{J}(\underline{\theta})=\frac{1}{2} \sum_{k=1}^{N}\left\|\underline{y}_{k}-\underline{y}_{k}(\underline{\theta})\right\|_{\left(\mathbf{Q}_{k}\right)^{-1}}^{2}$,

where the weighting matrix $\mathbf{Q} \in \mathbb{R}^{L \times L}$ is assumed to be positive definite

$\|a\|_{\left(\mathbf{Q}_{k}\right)^{-1}}^{2}=a^{\mathrm{T}}\left(\mathbf{Q}_{k}\right)^{-1} a, \forall a \in \mathbb{R}^{L}$.

Here $\underline{y}_{k} \in \mathbb{R}^{L}$ stands for the predicted values corresponding to the parameter vector $\underline{\theta}=\left[E, \nu, \rho, \eta_{1}, \eta_{2}, \alpha, \beta\right]^{\mathrm{T}} \in \mathbb{R}^{7}$ that contains the physical parameters of the heart surface and the weighting coefficients describing the boundary conditions.

In order to ensure the physical meaning of the model parameters, the physical constraints are incorporated in the calibration procedure. For fitting the model to the observations, only the parameters from the bounded intervals are considered. For example, the Young's modulus $E$ is positive. The Poisson's ratio is bounded by two theoretical limits: it must be greater or equal than 0 , and less than or equal to 0.5 .

\section{Physics-Based Tracking Approach}

The physics-based tracking approach proposed in this section allows the robust and efficient tracking of every point on the heart surface without any assumptions on the type of the heart's movement. The tracking process combines the reconstructed measurements with the information from the heart surface model in order to achieve a robust tracking. Below, a detailed description of this process is given.

Due to the fact that the obtained system equation (6) and the measurement equation (7) are linear, the Kalman filter ensures the best possible estimate for the system state characterizing the motion of heart surface. Assuming the process and the measurement noise as uncorrelated and by representing the phenomenon state as a Gaussian random vector $\underline{\boldsymbol{\xi}} \sim \mathcal{N}\left(\underline{\mu}_{k}^{e}, \mathbf{C}_{k}^{e}\right)$, this estimator recursively updates the state mean $\underline{\mu}_{k}^{e}$ and its covariance $\mathbf{C}_{k}^{e}$ in two steps. 


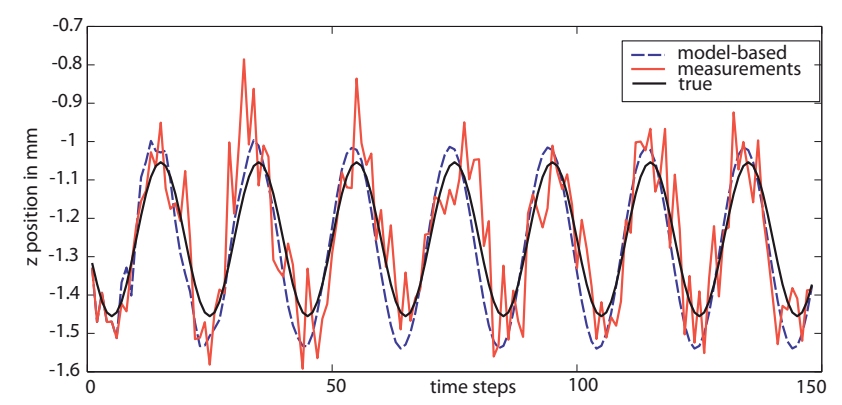

(a) Model-based prediction using ARX model.

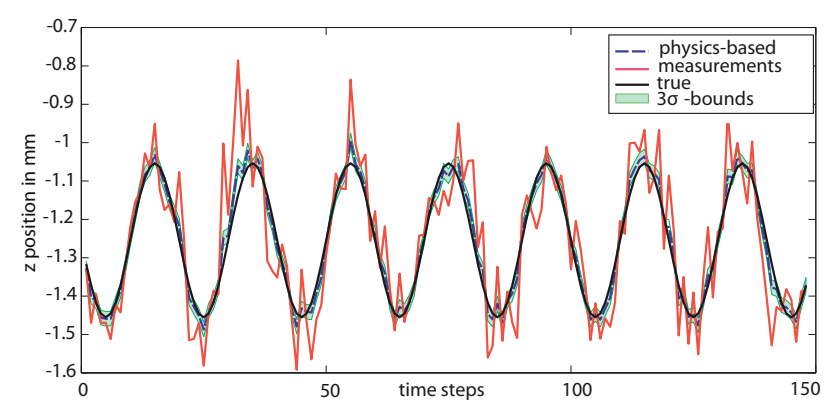

(b) Physics-based prediction.

Fig. 7 Simulated results for the comparison of the model-based and the physics-based estimation using noisy observations. The parameter of both models are detected considering the heart surface motion in all directions. The ARX model fits the noisy measurement data and its prediction deviates strongly from the true realization of the heart surface motion. The physics-based prediction fits the true values and thus, provides more accurate results since the physical characteristics of a system are considered.

In the prediction step or time update, the state and the covariance are projected forward to the next time step $k+1$ according to [28]

$\underline{\hat{\mu}}_{k+1}^{p}=\mathbf{A}_{k} \underline{\hat{\mu}}_{k}^{e}+\mathbf{B}_{k} \underline{\hat{p}}_{k}$,

$\hat{\mathbf{C}}_{k+1}^{p}=\mathbf{A}_{k} \mathbf{C}_{k}^{e} \mathbf{A}_{k}^{\mathrm{T}}+\mathbf{B}_{k} \mathbf{C}^{w} \mathbf{B}_{k}^{\mathrm{T}}$,

where $\underline{\mu}_{k}^{e}$ and $\underline{\mu}_{k+1}^{p}$ denote the mean of the estimated and predicted state, respectively. The state contains information about the 3D position of the measurement points $\underline{X}$.

If the reconstructed measurement point is in the uncertainty region of the predicted point it is accepted as a track or corresponding measurement of this point.

If the reconstructed measurements $\underline{\hat{y}}_{k}$ are accessible, the filter step or measurement update fuses the predicted state estimate with the measurement information according [28]

$\underline{\hat{\mu}}_{k}^{e}=\underline{\hat{\mu}}_{k}^{e}+\mathbf{K}_{k}\left(\underline{\hat{y}}_{k}-\mathbf{H}_{k} \underline{\mu}_{k}\right)$,

$\mathbf{C}_{k}^{e}=\left(\mathbf{I}-\mathbf{H}_{k} \mathbf{K}_{k}\right) \mathbf{C}_{k}^{p}$,

where

$\mathbf{K}_{k}=\mathbf{C}_{k}^{p} \mathbf{H}_{k}^{\mathrm{T}}\left(\mathbf{H}_{k} \mathbf{C}_{k}^{p} \mathbf{H}_{k}^{\mathrm{T}}+\mathbf{C}^{v}\right)^{-1}$.

Here, $\mathbf{I}$ denotes the identity matrix and $\mathbf{C}^{v}$ is the covariance matrix of the measurement noise. In case of occlusions, only the predicted information about the $3 \mathrm{D}$ position of the measurement points is given. In this way, the model-based estimation allows an efficient handling of occlusions by providing predicted estimates when no measurements exist.

\section{Evaluation}

This section evaluates the proposed physics-based tracking approach in simulations and experimental results. First, the performance of the physics-based heart surface motion model is highlighted in comparison to the standard autoregressive model with an exogenous input (ARX), which is proposed in [29] for a prediction of the heart motion. Second, after description of the experimental setup, an experiment on the pressure-regulated artificial heart is provided. The aim of the experiment is to test the flexibility of the initialization process and evaluate the prediction quality of the physicsbased tracking approach. Finally, the tracking algorithm is analyzed regarding its computational efficiency.

\subsection{Simulation}

In order to point out the impact of a physics-based estimation in comparison to the ARX model, we consider the example of estimating the z-displacement of a beating heart from noisy measurements. The ground truth of the heart motion is simulated by assuming that an accurate model of the process dynamics with known parameters is available. The true parameters of the physical model are Young's modulus $E=5 \mathrm{MPa}$, Poisson's ratio $\nu=0.28$, material density $\rho=100 \mathrm{gm}^{-3}$, damping constants $\eta_{1}=0.51 \mathrm{Ns}^{2} \mathrm{~m}^{-1}$, $\eta_{2}=0.32 \mathrm{Ns}^{2} \mathrm{~m}^{-1}$ and coefficients defining the boundary conditions $\alpha=0.1, \beta=0.9$. The pressure corrupted by a white zero-mean Gaussian noise with covariance $\sigma^{2}=$ $100 \mathrm{kPa}^{2}$ is assumed to be periodic with the frequency $0.2 \mathrm{~Hz}$ and amplitude $10000 \mathrm{kPa}$. The measurements of the heart surface motion on 30 measurement points are simulated by adding a white zero-mean Gaussian noise with covariance $\sigma^{2}=0.0081 \mathrm{~mm}^{2}$. The initial state is determined by solving the static equation describing the heart surface motion. The initial covariance matrix is defined as a diagonal matrix $\mathbf{C}_{k}^{e}=\operatorname{diag}\{0.1, \ldots, 0.1\} \mathrm{mm}^{2}$ with 360 entries corresponding to the number of the system states. The process and measurement noise are given by covariance matrices $\mathbf{C}^{w}$ and $\mathbf{C}^{v}$ with diagonal entries $100 \mathrm{kPa}^{2}$ and $0.0081 \mathrm{~mm}^{2}$ respectively.

A Kalman filter is used to compute state estimates by recursive prediction and filtering. The prediction horizon of one time step is used. For comparison of the physics-based approach with ARX model, the prediction error is defined as a second norm of the difference between the true and predicted position averaged over all time steps. For simplicity, the motion of only one point on the heart surface is considered. 
Table 1 Prediction Results

\begin{tabular}{|c|c|c|}
\hline methods & error in $\mathrm{mm}$ & standard deviation in $\mathrm{mm}$ \\
\hline measurements & 0.0782 & 0.0601 \\
model-based & 0.0558 & 0.0315 \\
physics-based & 0.0196 & 0.0154 \\
\hline
\end{tabular}

The prediction errors provided by both approaches are presented in Table 1. As presented in Fig. 7(b), the position of the heart surface predicted by the physics-based estimation closely resembles the true values. Since the physicsbased approach applies the physical constraints on the deformation of the heart surface motion in different directions, a physically correct and more accurate prediction is guaranteed, especially in a case of highly accurate physical model. In contrast to the physics-based model, the ARX model [30] of order 9 fits the noisy measurement data. Thereby, the predicted position of the heart surface deviates strongly from the true realization of the heart surface motion since the physical characteristics of the system are ignored. Therefore, if the measurements are corrupted by a high noise a physically incorrect and thus inaccurate prediction can be obtained.

\subsection{Experiment}

The performance of the proposed approach by estimating the state of a pressure-regulated artificial heart is evaluated in this section. After describing the experimental setup, we analyze the prediction accuracy. For this, we compare the predicted results with the measurements that were not considered by estimation. In this way, we show the performance of the physics-based tracking approach in handling of occlusions, when no measurement information is available.

\subsubsection{Experimental Setup}

The proposed tracking algorithm is running on a desktop PC with a Pentium IV $2.4 \mathrm{GHz}$ CPU and $512 \mathrm{MB}$ RAM. Image processing, reconstruction algorithms, and measurement-based tracking are implemented in $\mathrm{C}++$. The computation of the physical heart surface model and information fusion are overtaken by procedures running in Matlab. The performance analysis is accomplished on an image sequence stemming from a trinocular camera system. The motion of a pressureregulated artificial heart, depicted in Fig. 9, is measured in a laboratory. The pressure signal is periodic with the amplitude $100 \mathrm{hPa}$ and frequency $1.2 \mathrm{~Hz}$.

The motion of the stabilized beating heart, according to the measurement experiment performed in [31], is $0.59 \mathrm{~mm}$ in the lateral plane (x-y axis) and $2.1 \mathrm{~mm}$ out-of-plane (zaxis). For evaluation of the tracking algorithm, the motion of the pressure-regulated artificial heart shown in Fig. 9 is tracked. The amplitude of the artificial heart motion is $1.3 \mathrm{~mm}$ and $1.7 \mathrm{~mm}$ in $\mathrm{x}$ - and $\mathrm{y}$-direction, respectively, and $3.7 \mathrm{~mm}$ out-of-plane, as shown in Fig. 8, where estimated and measured motion of point 12 on the heart surface is depicted. Therefore, the motion of the artificial heart is assumed to be comparable to the real heart deformations.

For tracking the heart surface motion, the trinocular camera system is installed at a distance of $50 \mathrm{~cm}$ over the monitored operation area. The three cameras PIKE F-210C with resolution 1920 pixel $\times 1080$ pixel provide an accuracy of $0.2 \mathrm{~mm} / \mathrm{pixel}$ in $\mathrm{y}$-direction and $0.11 \mathrm{~mm} / \mathrm{pixel}$ in $\mathrm{x}$-direction of the image coordinate system [32]. The experimental setup is shown in Fig. 3, wherein the camera baselines are about $57 \mathrm{~cm}$, their focal length is about $35 \mathrm{~mm}$ and the field of view is $12.8 \mathrm{~cm} \times 17.02 \mathrm{~cm}$. The image size of every camera is reduced by cutting out of the defined regions of interest (ROI). The size of ROI is 417600 pixel, 453600 pixel, and 422100 pixel in the first, second and third camera views respectively. The cameras are calibrated using a multiple cameras calibration toolbox with a laser pointer [33]. The projection accuracy achieved by the calibration is 1.2 pixel. The acquired data are transfered via the communication protocol FireWire IEEE 1394b with a frame rate of about $30 \mathrm{~Hz}$. The cameras are software-triggered and synchronized. On the surface of the artificial heart simulator, the colored landmarks of $5 \mathrm{~mm}$ diameter, as shown in Fig. 6 , are manually attached. The centers of the landmarks are defined as measurement points. They are extracted with a subpixel accuracy by the segmentation algorithm, described in Section 2. It should be noted, that the precision of the tracking system depends on the amount of distributed measurement points. For this experiment, 26 artificial landmarks have been chosen.

\subsubsection{Initialization Process}

The initialization process is evaluated using an observation phase of two periods of the heart motion. The frame rate of the data acquisition is about $30 \mathrm{~Hz}$. In Fig. 9(a) the measurement points identified by main initialization process are shown. The loss of measurement information was caused by specular reflections. Further, the lost points are initialized by the measurement-based tracking running parallel to the physics-based tracking, as shown in Fig. 9(b). With the proposed initialization algorithm, additional landmarks may be introduced at any time. This flexibility of the initialization allows us to improve the precision of the physics-based tracking. Furthermore, it gives a surgeon the possibility to dynamically add or replace measurement points during surgery.

The initialized parameters of the heart surface model represented by the vector $\underline{\theta}=\left[E, \nu, \rho, \eta_{1}, \eta_{2}, \alpha, \beta\right]^{\mathrm{T}}$, are determined by the weighted least-squares approach according to equation (8). The obtained parameters are Young's modulus $E=24.9 \mathrm{MPa}$, Poisson's ratio $\nu=0.33$, material den- 

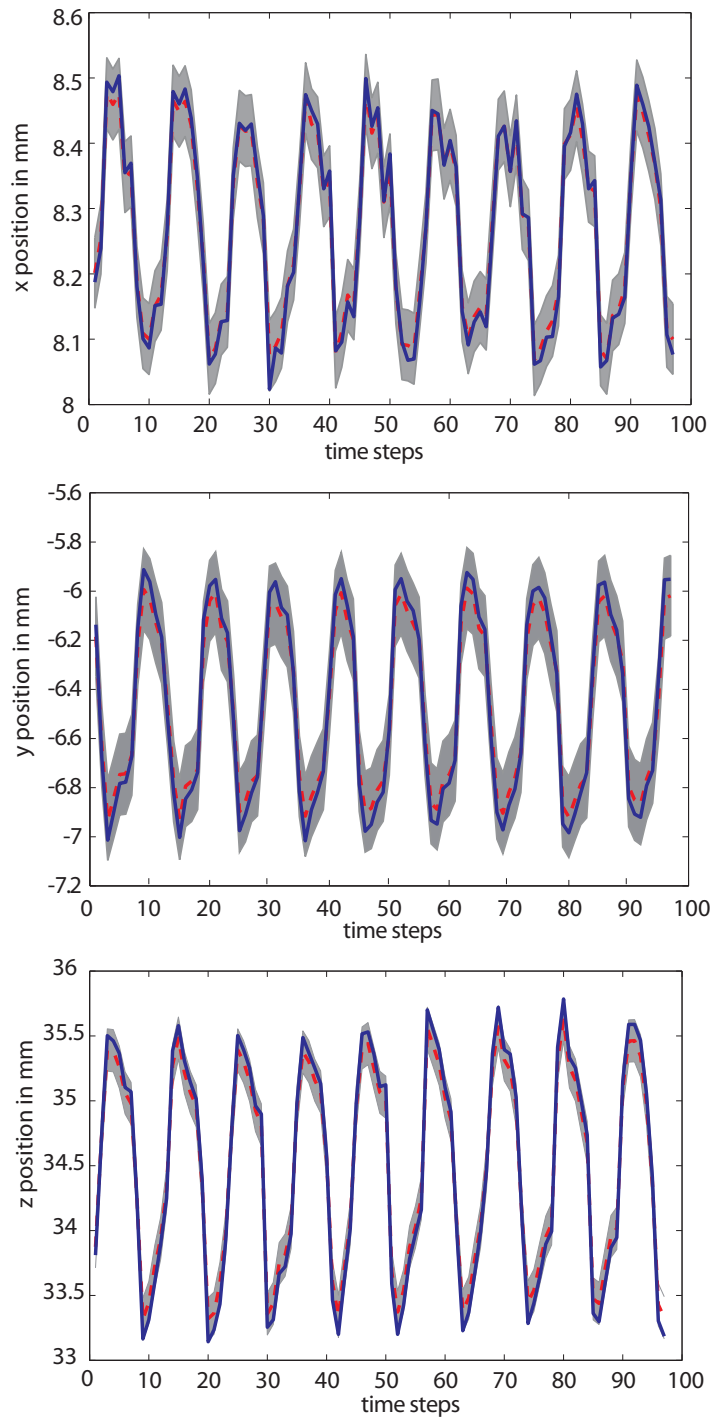

Fig. 8 Estimated solution (dashed line) and its $3 \sigma$-bounds (shaded region) for one measurement point in comparison to the measurements (solid line).

sity $\rho=1298.94 \mathrm{kgm}^{-3}$, damping constants $\eta_{1}=0.55 \mathrm{Ns}^{2} \mathrm{~m}^{-1}, \eta_{2}=0.028 \mathrm{Ns}^{2} \mathrm{~m}^{-1}$ and coefficients defining the boundary conditions $\alpha=0.49, \beta=0.44$.

\subsubsection{Tracking Approach}

The prediction quality of the proposed physics-based tracking approach is evaluated in the case of total occlusions. By comparing the estimated heart surface position with highly accurate observations in case of total occlusions, we determine, if the heart surface motion estimates are close to the measurements.

The accuracy of the $3 \mathrm{D}$ reconstruction is $0.26 \mathrm{~mm} \pm$ $0.12 \mathrm{~mm}$ according to the experimental evaluation. In order to evaluate the measurement system, a plane image of a calibration chess board is measured in different positions. This

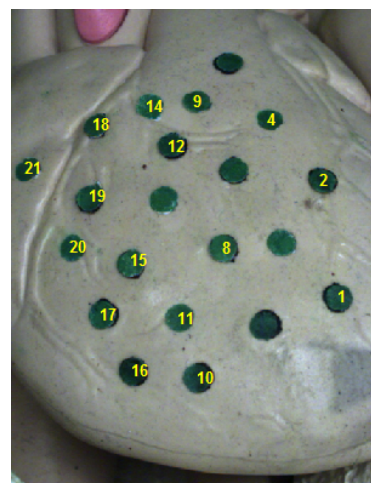

(a) Identified by main initialization process measurement points.

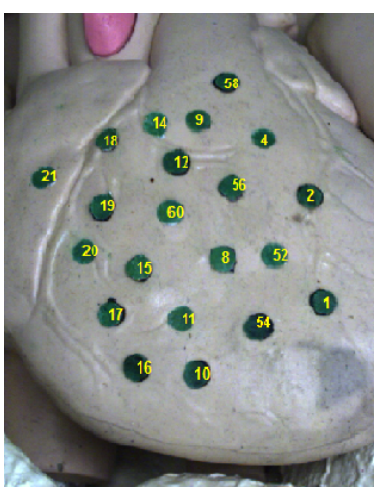

(b) Measurement points identified during physics-based tracking
Fig. 9 Initialization of physics-based tracking approach. The loss of measurement information depicted in Fig. 9(a) was caused by specular reflections. In Fig. 9(b) is shown that the lost points were successfully detected by the second initialization process

board consists of 10 squares with $10 \mathrm{~mm}$ sides. The value describing the accuracy of the reconstruction is determined by averaging the second norm of the difference between the reconstructed and true side lengths of the calibration board.

The performance of the tracking system in case of total occlusions is evaluated in comparison with the measurements, since the ground truth motion is unknown. For this purpose, the heart surface motion is measured by a trinocular camera system. Then, the heart movement is estimated using the acquired measurement data and information provided by the physical heart surface model. The total occlusion is simulated from time step 10 to time step 90 by ignoring all available measurements during estimation of the heart motion. In this case, the prediction provided by physics-based tracking approach is not corrected by the measurements and therefore represents the prediction accuracy of the tracking. The prediction error is defined as the second norm of the difference between the mean value of the predicted heart surface position and the $3 \mathrm{D}$ reconstruction based on priory obtained measurements.

The process and measurement noise are determined by covariance matrices $\mathbf{C}^{w}=\operatorname{diag}\{1.2, \ldots, 1.2\} \mathrm{kPa}^{2}$ and $\mathbf{C}^{v}=\operatorname{diag}\{0.14, \ldots, 0.14\} \mathrm{mm}^{2}$. The initial state is computed by solving the partial differential equation (1) in its static form. The initial state covariance matrix is assumed $\mathbf{C}_{k}^{e}=\operatorname{diag}\{3, \ldots, 3\} \mathrm{mm}^{2}$.

The physics-based prediction of the motion on one point in case of total occlusion is shown in Fig. 10. The prediction error of the physics-based tracking approach is defined as the second norm of the difference between the measurements and mean values of the predicted state. This error averaged over all time steps is $0.15 \mathrm{~mm}$. Thus, the presented results show that the accuracy of the heart surface motion reconstruction by means of physics-based prediction is in- 
side of the measurement accuracy. The main advantage of the physics-based tracking approach is that the long-term total occlusions can be handled. In this case, no measurement information about the heart surface motion is provided and thus, the approaches interpolating the heart motion can not be applied. Apparently, given an accurate and convergent model of the heart's dynamic, the physics-based tracking approach successfully copes with the total occlusions by predicting the heart surface motion under consideration of the pressure inside of the heart. Since the motion of the artificial heart is comparable with the real heart motion, the physicsbased tracking approach copes successfully with the heart deformations and can bridge total long-term occlusions.

\subsubsection{Computational time}

The speed of the physics-based tracking approach is measured by taking its average computational time over a set of 200 frames. The computational time per frame varies from $9.8 \mathrm{~ms}$ to $10.5 \mathrm{~ms}$ and is on average $10 \mathrm{~ms}(100 \mathrm{~Hz})$. This time includes the time needed for the computation of the heart model, the correspondence analysis, the 3D reconstruction, and the information fusion. Thus, a high computational efficiency of the physics-based tracking approach is achieved, so that the real-time requirement on a robotic surgery system is fulfilled.

The initialization procedure presented in Section 5 is tested on 184 frames. This allows data processing for 8 periods of heart movement. The average computational time for processing one frame is $4 \mathrm{~ms}$, which corresponds to the high processing frequency of $250 \mathrm{~Hz}$. This processing time represents the high computational efficiency of the measurement-based tracking algorithm.

The obtained experimental results prove the efficiency of the proposed tracking algorithm, which provides a precise estimation of the heart surface movement.

\section{Conclusion}

In this paper, a stochastic physics-based tracking approach for beating heart surgery has been proposed. It incorporates the physical heart surface model and an efficient reconstruction algorithm. The model does not imply constraints on the periodicity of the heart movement or out-of-plane deflection. Due to application of the meshless methods, the complexity of the physics-based modeling is significantly reduced. The reconstruction algorithm is efficient by incorporating the constraints provided by a physics-based prediction and a trinocular camera measurement system.

The principle advantage of the proposed method is that the physics-based tracking successfully copes with total longterm occlusions. In this case, the conventional interpolation methods cannot be applied, because no measurement information is available. In contrast to the standard model-based

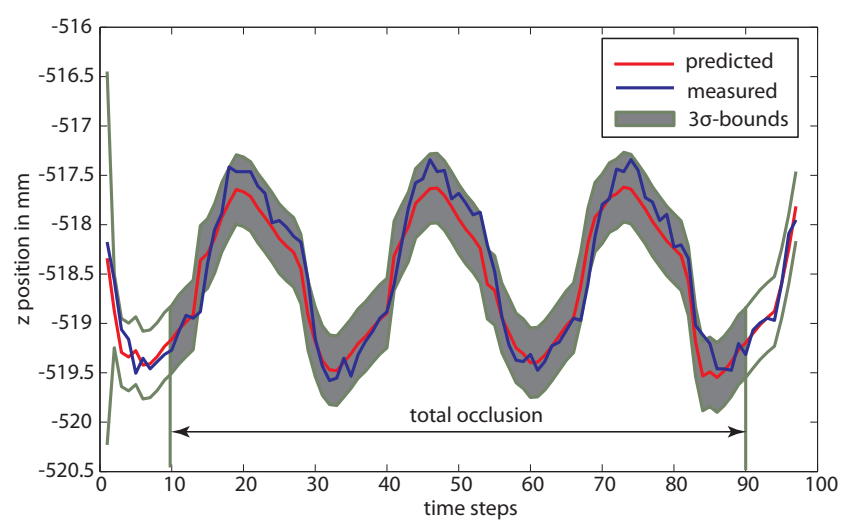

Fig. 10 Performance of the physics-based tracking in case of total occlusion occurring during time steps 10 to 90 . The physics-based state prediction is compared with the measurements, which were not considered in the estimation. The approach copes successfully with the total occlusion, when no measurement information is available.

methods, the physics-based tracking approach ensures a physically correct and more accurate prediction by considering the stochastic uncertainties and physical characteristics of the heart. The experimental results show the high performance of the tracking method.

In near the future a clinical evaluation of the proposed approach is envisaged. For this purpose, the proposed automatic initialization of the tracking will adapt the physical model to every patient. The tracking approach allows introduction of additional measurement points at any time. This gives a surgeon the flexibility to add, remove, or replace measurement points on the heart surface during surgery. Since the measurements provided by multi-camera system allows more reliable reconstruction, the possible integration of a trinocular camera system in an endoscope for application in minimally invasive surgery is planned.

Acknowledgements This work was partially supported by the German Research Foundation (DFG) within the Research Training Group RTG 1126 "Intelligent Surgery - Development of new computer-based methods for the future working environment in visceral surgery"

\section{References}

1. Chomiac, P., Bose, S., Page, M.: Beating Heart Bypass Surgery (2001)

2. Jacobs, S., Holzhey, D., Kiaii, B.B., Onnasch, J.F., Walther T., Mohr, F.W., Falk, V.: Limitations for Manual and Telemanipulator-Assisted Motion Tracking - Implications for Endoscopic Beating-Heart Surgery. The Annals of Thoracic Surgery 76, 2029-2036 (2003)

3. Falk, V.: Manual Control and Tracking - A Human Factor Analysis Relevant for Beating Heart Surgery. The Annals of Thoracic Surgery 74, 624-628 (2002)

4. Çavuşoğlu, M.C., Rotella, J., Newman, W.S., Choi, S., Ustin, J. Sastry, S.S.: Control Algorithms for Active Relative Motion Cancelling for Robotic Assisted Off-Pump Coronary Artery Bypass Graft Surgery. In: Proceedings of the 12th International Conference on Advanced Robotics (ICAR 2005), pp. 431-436. Seattle, USA (2005) 
5. Nyquist, H.: Certain Topics in Telegraph Transmission Theory. Transactions of the American Institute of Electrical Engineers 47, 617-644 (1928)

6. Nakamura, Y., Kishi, K., Kawakami, H.: Heartbeat Synchronization for Robotic Cardiac Surgery. In: Proceedings of the IEEE International Conference on Robotics and Automation (ICRA 2001), pp. 2014-2019. Seoul, Korea (2001)

7. Stoyanov, D., Mylonas, G.P., Deligianni, F., Darzi, A., Yang, G.Z.: Soft-Tissue Motion Tracking and Structure Estimation for Robotic Assisted MIS Procedures. In: Proceedings of the Medical Image Computing and Computer Assisted Interventions (MICCAI 2005), vol. 2, pp. 139-146 (2005)

8. Ramey, N.A., Corso, J.J., Lau, W.W., Burschka, D., Hager, G.D.: Real-time 3D Surface Tracking and Its Applications. In: Proceedings of the 2004 Conference on Computer Vision and Pattern Recognition Workshop (CVPRW 2004) (2004)

9. Lau, W.W., Ramey, N.A., Corso, J.J., Thakor, N.V., Hager, G.D.: Stereo-Based Endoscopic Tracking of Cardiac Surface Deformation. In: Proceedings of the International Conference on Medical Image Computing and Computer-Assisted Intervention (MICCAI 2004), pp. 494-501 (2004)

10. Richa, R., Poignet, P., Liu, C.: Efficient 3D Tracking for Motion Compensation in Beating Heart Surgery. In: Proceeding of the Medical Image Computing and Computer-Assisted Intervention (MICCAI 2008), vol. 5242, pp. 684-691 (2008)

11. Sauvée, M., Poignet, P., Triboulet, J., Dombre, E., Malis, E., Demaria, R.: 3D Heart Motion Estimation Using Endoscopic Monocular Vision System. In: Proceeding of the IFAC Symposium on Modeling and Control in Biomedical Systems, pp. 1-6 (2006)

12. Noce, A., Triboulet, J., Poignet, P.: Efficient Tracking of the Heart Using Texture. In: Proceedings of the Annual International Conference of the IEEE Engineering in Medicine and Biology Society (EMBS 2007), pp. 4480-4483. Lyon, France (2007)

13. Sauvée, M., Noce, A., Poignet, P., Triboulet, J., Dombre, E.: Three-dimensional heart motion estimation using endoscopic monocular vision system: From artificial landmarks to texture analysis. In: Proceeding of the IFAC Symposium on Modeling and Control in Biomedical Systems, 3, vol. 2, pp. 199-207 (2007)

14. Ortmaier, T., Groeger, M., Boehm, D.H., Falk, V., Hirzinger, G.: Motion Estimation in Beating Heart Surgery. IEEE Transactions on Biomedical Engineering 52(10), 1729-1740 (2005)

15. Özkan Bebek, Çavuşoğlu, M.C.: Intelligent Control Algorithms for Robotic-Assisted Beating Heart Surgery. IEEE Transactions on Robotics 23(3), 468-480 (2007)

16. Richa, R., Poignet, P., Liu, C.: Deformable Motion Tracking of the Heart Surface. In: Proceedings of the 2008 IEEE International Conference on Intelligent Robots and Systems (IROS 2008) (2008)

17. Bader, T., Wiedemann, A., Roberts, K., Hanebeck, U.D.: Model-based Motion Estimation of Elastic Surfaces for Minimally Invasive Cardiac Surgery. In: Proceedings of the 2007 IEEE International Conference on Robotics and Automation (ICRA 2007), pp. 2261-2266. Rome, Italy (2007). DOI 10.1109/ROBOT.2007.363656
18. Bogatyrenko, E., Hanebeck, U.D., Szabo, G.: Heart Surface Motion Estimation Framework for Robotic Surgery Employing Meshless Methods. In: Proceedings of the 2009 IEEE/RSJ International Conference on Intelligent Robots and Systems (IROS 2009) (2009)

19. Shi, P., Liu, H.: Stochastic Finite Element Framework for Cardiac Kinematics Function and Material Property Analysis. In: Proceedings of the Medical Image Computing and Computer-Assisted Intervention (MICCAI 2002), pp. 634-641 (2002)

20. Sermesant, M., Moireau, P., Camara, O., Sainte-Marie, J., Andriantsimiavona, R., Cimrman, R., Hill, D., Chapelle, D., Razavi, R.: Cardiac Function Estimation from MRI using a Heart Model and Data Assimilation: Advances and Difficulties. In: Functional Imaging and Modeling of the Heart, vol. 10, pp. 642-656 (2006)

21. Hartley, R., Zisserman, A.: Multiple View Geometry in Computer Vision. Cambridge University Press, Cambridge (2000)

22. Mulligan, J., Isler, V., Daniilidis, K.: Trinocular Stereo: a RealTime Algorithm and Its Evaluation. International Journal of Computer Vision 27, 51-61 (2001)

23. Zahorec, R., Holoman, M.: Transatrial Access for Left Atrial Pressure Monitoring in Cardiac Surgery Patients. European Journal of Cardio-Thoracic Surgery 11, 379-380 (1997)

24. Atluri, S.N., Han, Z.D., Liu, H.T.: Meshless Local PetrovGalerkin (MLPG) Mixed Collocation Method for Elasticity Problems. Tech Science Press, CMES 14(3), pp.141-152 (2006)

25. Liu, G.R.: Mesh Free Methods: Moving Beyond the Finite Element Methods. CRC Press (2003)

26. Liu, G.R.: An Introduction to Meshfree Methods and Their Programming. Springer (2005)

27. Uciński, D.: Optimal Measurement Methods for Distributed Parameter System Identification (2005)

28. Kalman, R.E.: A New Approach to Linear Filtering and Prediction Problems. Transactions of the ASME Journal of Basic Engineering 82, 35-45 (1960)

29. Yuen, S.G., Novotny, P.M., Howe, R.D.: Quasiperiodic Predictive Filtering for Robot-Assisted Beating Heart Surgery. In: Proceeding of the IEEE International Conference on Robotics and Automation (ICRA 2008), pp. 3875-3880. Pasadena, CA, USA (2008)

30. Box, G., Jenkins, G.M., Reinsel, G.C.: Time Series Analysis: Forecasting and Control. Prentice-Hall (1994)

31. Cattina, P., Daveb, H., Gruenenfelder, J., Szekelya, G., Turin, M., Zuend, G.: Trajectory of Coronary Motion and Its Significance in Robotic Motion Cancellation. European Journal of Cardiothoracic Surgery 25, 786-790 (2004)

32. Allied Vision Technologies GmbH: PIKE Technical Manual V4.2.0 (2009)

33. Svoboda, T., Martinec, D., Pajdla, T.: A Convenient Multi-Camera Self-Calibration for Virtual Environments. Presence: Teleoperators and Virtual Environments 14(4), 1-26 (2005) 